\title{
Nonleptonic kaon decays: theory vs. experiment
}

\author{
C.Cheshkov \\ Joint Institute for Nuclear Research, Dubna, Russian Federation
}

October 27, 2018

\begin{abstract}
A review of the present experimental status of the rates and the Dalitz-plot slope parameters in CP conserving $K \rightarrow 2 \pi$ and $K \rightarrow$ $3 \pi$ decays is given. The corresponding isospin amplitudes have been determined by a common fit to the recent experimental data and have been used as an input to a consequent fit based on the constraints from $O\left(p^{4}\right)$ chiral lagrangian. It has been found that the constraints of the chiral fit are well satisfied by the experimental data, allowing to estimate the weak coupling constants and to predict the quadratic amplitudes in $K \rightarrow 3 \pi$ decays and the one-loop strong rescattering phases. The consistency of the obtained weak coupling constants with weak resonance models has been also considered.
\end{abstract}




\section{Introduction}

Quantum Chromodynamics (QCD) is the established theory for describing strong quark-quark interactions. However, the theoretical study of kaon decays is hindered by the nonperturbative behavior of QCD in the low-energy domain. One of the approaches successfully applied to the study of lowenergy processes is chiral perturbation theory (ChPT) [1, 2]. It is based on the chiral symmetry properties of QCD and the concept of the effective field theory. An important point in the application of $\mathrm{ChPT}$ is the existence of coupling constants which can not be calculated from first principles and therefore must be extracted phenomenologically from the experimental data.

In the present paper we report an update of the analysis of $\mathrm{CP}$ conserving $K \rightarrow 2 \pi$ and $K \rightarrow 3 \pi$ decays, performed in [3]. The update is caused by necessity to take into account the recent measurements of $\Gamma\left(K_{S} \rightarrow \pi^{+} \pi^{-} \pi^{0}\right)$ and the Dalitz plot parameters in $K \rightarrow 3 \pi$ decays, carried out by the CPLEAR [4], the HYPERON [5] and the NA48 [6] collaborations.

In section 2 we present the results from a phenomenological fit of $K \rightarrow 2 \pi$ and $K \rightarrow 3 \pi$ isospin amplitudes to the recent experimental data. In section 3 we consider the estimation of the isospin amplitudes in terms of $O\left(p^{4}\right)$ chiral lagrangian and summarize the results from a fit of the weak coupling constants. In section 4 we briefly discuss the consistency of the obtained results with weak resonance models. Section 5 contains some concluding remarks.

\section{Phenomenological fit}

Using the usual isospin decomposition CP conserving $K \rightarrow 2 \pi$ amplitudes can be expressed in the following wayt:

$$
\begin{aligned}
A\left(K^{0} \rightarrow \pi^{+} \pi^{-}\right) & =-\frac{1}{\sqrt{3}} A_{0}+\frac{1}{\sqrt{6}} A_{2} \\
A\left(K^{0} \rightarrow \pi^{0} \pi^{0}\right) & =\frac{1}{\sqrt{3}} A_{0}+\sqrt{\frac{2}{3}} A_{2} \\
A\left(K^{+} \rightarrow \pi^{+} \pi^{0}\right) & =\frac{\sqrt{3}}{2} A_{2}
\end{aligned}
$$

where $A_{0}=i a_{1 / 2} e^{i \delta_{0}}$ and $A_{2}=-i a_{3 / 2} e^{i \delta_{2}}$ represent transitions with $\Delta I=$ $1 / 2,3 / 2$, respectively.

Due to the small available phase space in $K \rightarrow 3 \pi$ decays their amplitudes can be expanded in powers of Dalitz plot variables $u$ and $v$ :

\footnotetext{
${ }^{1}$ assuming $\Delta I \leq 3 / 2$
} 


$$
\begin{aligned}
A\left(K_{L} \rightarrow \pi^{+} \pi^{-} \pi^{0}\right) & =\left(\alpha_{1}+\alpha_{3}\right)-\left(\beta_{1}+\beta_{3}\right) u \\
& +\left(\zeta_{1}-2 \zeta_{3}\right)\left(u^{2}+v^{2} / 3\right)+\left(\xi_{1}-2 \xi_{3}\right)\left(u^{2}-v^{2} / 3\right) \\
A\left(K_{L} \rightarrow \pi^{0} \pi^{0} \pi^{0}\right) & =-3\left(\alpha_{1}+\alpha_{3}\right)-3\left(\zeta_{1}-2 \zeta_{3}\right)\left(u^{2}+v^{2} / 3\right) \\
A\left(K^{+} \rightarrow \pi^{+} \pi^{+} \pi^{-}\right) & =\left(2 \alpha_{1}-\alpha_{3}\right)+\left(\beta_{1}-\beta_{3} / 2+\sqrt{3} \gamma_{3}\right) u \\
& +2\left(\zeta_{1}+\zeta_{3}\right)\left(u^{2}+v^{2} / 3\right)-\left(\xi_{1}+\xi_{3}-\xi_{3}^{\prime}\right)\left(u^{2}-v^{2} / 3\right) \\
A\left(K^{+} \rightarrow \pi^{0} \pi^{0} \pi^{+}\right) & =-\left(\alpha_{1}-\alpha_{3} / 2\right)+\left(\beta_{1}-\beta_{3} / 2-\sqrt{3} \gamma_{3}\right) u \\
& -\left(\zeta_{1}+\zeta_{3}\right)\left(u^{2}+v^{2} / 3\right)-\left(\xi_{1}+\xi_{3}+\xi_{3}^{\prime}\right)\left(u^{2}-v^{2} / 3\right) \\
A\left(K_{S} \rightarrow \pi^{+} \pi^{-} \pi^{0}\right) & =(2 / \sqrt{3}) \gamma_{3} v-(4 / 3) \xi_{3}^{\prime} u v
\end{aligned}
$$

where

$$
u=\frac{s_{3}-s_{0}}{M_{\pi^{ \pm}}^{2}}, v=\frac{s_{2}-s_{1}}{M_{\pi^{ \pm}}^{2}}, s_{i}=\left(P_{K}-P_{\pi_{i}}\right)^{2}, s_{0}=\frac{\left(s_{1}+s_{2}+s_{3}\right)}{3}
$$

and $P_{K}, P_{\pi_{i}}$ are the four-momenta of the kaon and $i$-the pion. The subscripts 1 and 3 in (2) correspond to $\Delta I=1 / 2,3 / 2$ transitions, respectively. Neglecting the strong rescattering final state phases and assuming $\mathrm{CP}$ invariance all the isospin amplitudes $\alpha_{1}, \alpha_{3}, \beta_{1}, \beta_{3}, \gamma_{3}, \zeta_{1}, \zeta_{3}, \xi_{1}, \xi_{3}$ and $\xi_{3}^{\prime}$ are real.

Until now the experimentally measured event distributions for CP conserving $K \rightarrow 3 \pi$ decays have been analyzed in terms of the Dalitz plot slopes $g, h, k$ :

$$
|A(K \rightarrow 3 \pi)|^{2} \sim 1+g u+h u^{2}+k v^{2} .
$$

The recent experimental data [0] on the decay widths and the Dalitz plot slopes are reported in Table $\mathbb{1}$.

The phenomenological fit of $K \rightarrow 3 \pi$ isospin amplitudes to the measured decay widths and Dalitz plot slopes was introduced in [8] and was significantly improved in [3] by including the $\Delta I=3 / 2$ quadratic amplitudes $\zeta_{3}, \xi_{3}$ and $\xi_{3}^{\prime}$. We have redone the fit by adding the measurements of $\Gamma\left(K_{s} \rightarrow \pi^{+} \pi^{-} \pi^{0}\right)$ and the quadratic Dalitz plot slope $k$ in $K^{+} \rightarrow \pi^{0} \pi^{0} \pi^{+}$. The amplitudes $a_{1 / 2}, a_{3 / 2}$ and the relative phase $\left(\delta_{2}-\delta_{0}\right)$ between them have been evaluated using only the measured $K \rightarrow 2 \pi$ decay widths. The results for the isospin amplitudes are shown in the first column of Table 2. Due to small isospin breaking effects the obtained value of $-64.6^{\circ}$ for the relative phase between $a_{3 / 2}$ and $a_{1 / 2}$ are not reliable and has not been used in the further analysis. The relatively high value of $\chi^{2}$ of the fit is due entirely to an inconsistency of

\footnotetext{
${ }^{2}$ index 3 refers to the "odd" pion

${ }^{3}$ We have included the value of $\left(-6.1 \pm 0.9_{\text {stat }} \pm 0.5_{\text {syst }}\right) \times 10^{-3}$ for the quadratic Dalitz plot slope parameter $h$ in the $K_{L} \rightarrow 3 \pi^{0}$ decay, measured by the NA48 collaboration [6]
} 
the precisely measured $K_{L} \rightarrow 3 \pi$ and $K^{ \pm} \rightarrow 3 \pi$ decay widths. Contrariwise, all the measured Dalitz plot slopes and $\Gamma\left(K_{S} \rightarrow \pi^{+} \pi^{-} \pi^{0}\right)$ seems to be quite consistent.

We have also tried to extract the strong rescattering phase of the amplitudes $\beta$ from the available data. In order to do this one have to replace the real amplitudes $\beta$ in (2) with

$$
\beta \rightarrow \beta e^{i \delta_{\beta}} \approx \beta\left(1+i \delta_{\beta}\right)
$$

where $\delta_{\beta}$ is real [9]. Since the phases of the constant and quadratic amplitudes are expected to be considerably smaller than $\delta_{\beta}$ [10], they have been neglected. The phase $\delta_{\beta}$ has been introduced into the phenomenological fitting procedure as a freely varying parameter. As a result we have obtained $\left|\delta_{\beta}\right|<0.3(68 \% \mathrm{CL})$ while the isospin amplitudes have remained almost unchanged.

\section{Theoretical estimations within $O\left(p^{4}\right)$ ChPT}

To the lowest order $O\left(p^{2}\right)$, CP conserving weak chiral Lagrangian is a sum of $\left(8_{L}, 1_{R}\right)$ and $\left(27_{L}, 1_{R}\right)$ operators [11]:

$$
\mathcal{L}_{W}^{(2)}=c_{2} \operatorname{Tr} \lambda_{6} L_{\mu} L^{\mu}+c_{3} t_{i k}^{j l}\left(\operatorname{Tr} Q_{j}^{i} L_{\mu}\right)\left(\operatorname{Tr} Q_{l}^{k} L^{\mu}\right),
$$

where $\left(Q_{j}^{i}\right)_{a b}=\delta_{i b} \delta_{j a}$ are the projectors in flavor space, $\lambda_{6}=Q_{3}^{2}+Q_{2}^{3}, t_{i k}^{j l}$ are defined to select the 27-plet part of the interaction, $L_{\mu}=i U^{\dagger} \partial_{\mu} U$ is the left-handed weak current, $U=e^{\left(i \Phi / F_{\pi}\right)}, F_{\pi}=92.4 \mathrm{MeV}$ is the pion decay constant and $\Phi$ is the pseudoscalar meson $S U(3)$ matrix:

$$
\Phi=\sqrt{2}\left(\begin{array}{ccc}
\frac{\pi^{0}}{\sqrt{2}}+\frac{\eta}{\sqrt{6}} & -\pi^{+} & -K^{+} \\
\pi^{-} & -\frac{\pi^{0}}{\sqrt{2}}+\frac{\eta}{\sqrt{6}} & -K^{0} \\
K^{-} & -\bar{K}^{0} & -\frac{2 \eta}{\sqrt{6}}
\end{array}\right) .
$$

The lowest order chiral Lagrangian corresponds to "tree" diagrams which contain one weak vertex and gives the following contributions to the $\Delta I=$ 1/2 isospin amplitudes [3]:

$$
\begin{aligned}
\Re e A_{0} & =\frac{\sqrt{6}}{F_{\pi}^{2} F_{K}}\left(M_{K}^{2}-M_{\pi}^{2}\right)\left(c_{2}-\frac{2}{3} c_{3}\right) \\
\Re e \alpha_{1} & =\frac{1}{3 F_{\pi}^{3} F_{K}} M_{K}^{2}\left(c_{2}-\frac{2}{3} c_{3}\right) \\
\Re e \beta_{1} & =\frac{-1}{F_{\pi}^{3} F_{K}} M_{\pi}^{2}\left(c_{2}-\frac{2}{3} c_{3}\right)
\end{aligned}
$$

and to the $\Delta I=3 / 2$ ones: 


$$
\begin{aligned}
\Re e A_{2} & =\frac{-20}{\sqrt{3} F_{\pi}^{2} F_{K}}\left(M_{K}^{2}-M_{\pi}^{2}\right) c_{3} \\
\Re e \alpha_{3} & =\frac{20^{3} F_{K}}{9 F_{K}^{3} F_{3}^{2}} c_{3} \\
\Re e \beta_{3} & =\frac{5}{3 F_{\pi}^{3} F_{K}} \frac{M_{\pi}^{2}\left(5 M_{K}^{2}-14 M_{\pi}^{2}\right)}{\left(M_{K}^{2}-M_{\pi}^{2}\right)} c_{3} \\
\Re e \gamma_{3} & =\frac{-15}{2 \sqrt{3} F_{\pi}^{3} F_{K}} \frac{M_{\pi}^{2}\left(3 M_{K}^{2}-2 M_{\pi}^{2}\right)}{\left(M_{K}^{2}-M_{\pi}^{2}\right)} c_{3}
\end{aligned}
$$

Thus the coupling constants $c_{2}$ and $c_{3}$ of the $\Delta I=1 / 2,3 / 2$ operators are determined by the two well-measured $K \rightarrow 2 \pi$ isospin amplitudes $a_{1 / 2}$ and $a_{3 / 2}$. $S U(3)$ symmetry breaking is taken into account by fixing the kaon decay constant $F_{K}$ to its physical value of $113 \mathrm{MeV}$.

Now lets consider the corrections to the isospin amplitudes due to the one-loop contributions from $\mathcal{L}_{S}^{(2)} \times \mathcal{L}_{W}^{(2)}$ and the tree-level contributions from both $O\left(p^{4}\right)$ weak and strong chiral lagrangians. Following the analysis in [3, 12], we have factorized $K \rightarrow 2 \pi$ and $K \rightarrow 3 \pi$ isospin amplitudes in the following way:

$$
A_{i}=A_{i}^{(2)}+A_{i}^{(4)}
$$

where $A_{i}^{(2)}$ are the lowest order values defined by equations (8), (9) and $A_{i}^{(4)}$ are the corresponding next-to-lowest corrections. Furthermore, the latter can be decompressed as:

$$
A_{i}^{(4)}=A_{i}^{\text {loop }}(\mu)+A_{i}^{\text {wk.ct. }}(\mu)+A_{i}^{\text {st.ct. }}(\mu),
$$

where $A_{i}^{\text {loop }}(\mu), A_{i}^{\text {wk.ct. }}(\mu), A_{i}^{\text {st.ct. }}(\mu)$ are the one-loop contributions and the tree-level contributions from the counterterms of $O\left(p^{4}\right)$ weak and strong lagrangians, respectively. The parameter $\mu$ is the renormalization scale on which, in principle, $A_{i}^{(4)}$ must be independent.

Since the one-loop contributions $A_{i}^{\text {loop }}(\mu)$ arise from the lowest order weak lagrangian, they are proportional to the coupling constants $c_{2}$ and $c_{3}$ and are conveniently expressed in terms of the so-called reduced amplitudes $a_{i}^{(8)}$ and $a_{i}^{(27)}$ :

$$
A_{i}^{\text {loop }}=\left(c_{2} / F_{\pi}^{2}\right) a_{i}^{(8)}+\left(c_{3} / F_{\pi}^{2}\right) a_{i}^{(27)}
$$

For the calculation of the one-loop contributions we have used the values of the reduced amplitudes for $\mu=M_{\eta}$ obtained in [3] and presented in Table 3.

As it was shown in [3, 12], without external fields and neglecting corrections of order $M_{\pi}^{2} / M_{K}^{2}$ the contributions from weak counterterms can be expressed in terms of only 7 linear combinations $K_{i}, i=(1 \div 7)$, of weak cou-

\footnotetext{
${ }^{4}$ the explicit form of $K_{i}$ can be found in [3]
} 
pling constants. On the other side, the contributions from the counterterms of the strong lagrangian are described by 5 out of 10 well-known coupling constants $L_{i}, i=(1 \div 5)$ (Table 4) [2, 13]. The counterterm contributions to the isospin amplitudes are:

$$
\begin{aligned}
& \Re e A_{0}=\frac{-2 \sqrt{2}}{3 \sqrt{3} F_{\pi}^{2} F_{K}} M_{K}^{4}\left(K_{1}+K_{4}\right) \\
& \Re e A_{2}=\frac{-20}{3 \sqrt{3} F_{\pi}^{2} F_{K}} M_{K}^{4} K_{4} \\
& \Re e \alpha_{1}=\frac{-2}{27 F_{\pi}^{3} F_{K}} M_{K}^{4}\left[\left(K_{1}+K_{4}-K_{2}+2 K_{5}\right)\right. \\
&\left.+\frac{4\left(3 c_{2}-2 c_{3}\right)}{F_{\pi}^{2}}\left(4 L_{1}+4 L_{2}+2 L_{3}\right)\right] \\
& \Re e \alpha_{3}=\frac{20}{27 F_{\pi}^{3} F_{K}} M_{K}^{4}\left[\left(K_{4}+2 K_{5}\right)\right. \\
&\left.-\frac{8 c_{3}}{F_{\pi}^{2}}\left(4 L_{1}+4 L_{2}+2 L_{3}\right)\right] \\
& \Re e \beta_{1}=\frac{-1}{9 F_{\pi}^{3} F_{K}} M_{K}^{2} M_{\pi}^{2}\left[\left(-2 K_{1}-2 K_{4}+K_{3}+\frac{16}{27} K_{5}+\frac{35}{27} K_{6}-\frac{13}{9} K_{7}\right)\right. \\
&\left.+\frac{8\left(3 c_{2}-2 c_{3}\right)}{F_{\pi}^{2}}\left(-2 L_{1}+L_{2}-L_{3}+12 L_{4}\right)\right] \\
& \Re e \beta_{3}=\frac{5}{18 F_{\pi}^{3} F_{K}} \frac{M_{K}^{4} M_{\pi}^{2}}{\left(M_{K}^{2}-M_{\pi}^{2}\right)}\left[\left(10 K_{4}+4 K_{6}+3 K_{7}\right)\right. \\
&\left.+\frac{64 c_{3}}{F_{\pi}^{2}}\left(2 L_{1}-L_{2}+L_{3}-12 L_{4}\right)\right] \\
& \Re e \gamma_{3}=\frac{-5}{4 \sqrt{3} F_{\pi}^{3} F_{K}} \frac{M_{K}^{4} M_{\pi}^{2}}{\left(M_{K}^{2}-M_{\pi}^{2}\right)}\left[\left(6 K_{4}+K_{7}\right)\right. \\
&\left.+\frac{8 c_{3}}{F_{K}^{2}} \frac{M_{\pi}^{2}}{M_{K}^{2}}\left(-L_{3}+12 L_{4}+6 L_{5}\right)\right] \\
& \Re e \zeta_{1}=\frac{-1}{6 F_{\pi}^{3} F_{K}} M_{\pi}^{4}\left[\left(K_{2}-2 K_{5}\right)\right. \\
&\left.-\frac{8\left(3 c_{2}-2 c_{3}\right)}{F_{\pi}^{2}}\left(2 L_{1}+2 L_{2}+L_{3}\right)\right] \\
& \Re e \zeta_{3}=\frac{5}{3 F_{\pi}^{3} F_{K}} M_{\pi}^{4}\left[K_{5}\right. \\
&\left.-\frac{8 c_{3}^{3}}{F_{\pi}^{2}}\left(2 L_{1}+2 L_{2}+L_{3}\right)\right] \\
& \Re e \xi_{1}=\frac{-1}{6 F_{\pi}^{3} F_{K}} M_{\pi}^{4}\left[\left(K_{3}+\frac{16}{27} K_{5}+\frac{35}{27} K_{6}-\frac{13}{9} K_{7}\right)\right. \\
&\left.-\frac{8\left(3 c_{2}-2 c_{3}\right)}{F_{\pi}^{2}}\left(2 L_{1}-L_{2}+L_{3}\right)\right] \\
& \Re e \xi_{3}=\frac{-5}{24 F_{\pi}^{3} F_{K}} M_{\pi}^{4}\left[\left(4 K_{6}+3 K_{7}\right)\right. \\
&\left.+\frac{64 c_{3}}{F_{\pi}^{2}}\left(2 L_{1}-L_{2}+L_{3}\right)\right] \\
&-\frac{15}{8 F_{\pi}^{3} F_{K}} M_{\pi}^{4}\left[K_{7}\right. \\
&\left.F_{K}^{2} \frac{M_{\pi}^{2}}{M_{K}^{2}} L_{3}\right] \\
& \xi_{3}^{\prime} \\
& \Re_{3}
\end{aligned}
$$

Using the twelve isospin amplitudes in the first column of Table 2 and requiring that all the nine relative phases of $K \rightarrow 3 \pi$ amplitudes be zero within errors of $15^{\circ}$ (compatible with the upper bound of $\left|\delta_{\beta}\right|$ ), we have fitted the coupling constants $c_{2}, c_{3}$ and the counterterms $K_{i}$. The fitting procedure have shown that $K_{1}\left(K_{4}\right)$ is strongly correlated(anticorrelated) with $c_{2}\left(c_{3}\right)$. Thus, neglecting small terms of order $M_{\pi}^{2} / M_{K}^{2}$ in (8) and (9), the coupling constants $K_{1}$ and $K_{4}$ can be safely absorbed by a proper redefinition of the coupling constants $c_{2}$ and $c_{3}$ : 


$$
c_{2} \rightarrow c_{2}^{\prime}=c_{2}-\frac{2}{9} M_{K}^{2} K_{1}, \quad c_{3} \rightarrow c_{3}^{\prime}=c_{3}+\frac{1}{3} M_{K}^{2} K_{4} .
$$

In this way, the contributions $A_{i}^{(2)}, A_{i}^{\text {loop }}$ and $A_{i}^{\text {st.ct. }}$ are expressed in terms of $c_{2}^{\prime}$ and $c_{3}^{\prime}$ while the weak counterterm contributions $A_{i}^{\text {wk.ct. }}$ are determined by $K_{2}, K_{3}, K_{5}, K_{6}$ and $K_{7}$. The results of the fit are summarized in Table 5 and in the second column of Table 2. Obviously, the experimentally measured isospin amplitudes are in a excellent agreement within the applied chiral scheme. Moreover, the weak contributions of the quadratic amplitudes $\zeta_{1}$, $\zeta_{3}, \xi_{1}, \xi_{3}, \xi_{3}^{\prime}$ are proportional to the weak contributions of the constant and linear amplitudes and therefore are independent of the weak couplings $K_{i}$. Thus, the good consistency between the measured and predicted values of the quadratic amplitudes justifies the applicability of the chiral approach at $O\left(p^{4}\right)$.

Based on the fitted values of $c_{2}^{\prime}, c_{3}^{\prime}$ and $K_{i}$ and taking into account the imaginary parts of the one-loop contributions from Table 3, one can predict the phases of $K \rightarrow 3 \pi$ amplitudes. The obtained phases of the constant and linear amplitudes are given in Table 6. The imaginary parts of the transition amplitudes were expanded in Dalitz plot variables and calculated numerically. Therefore, considering the values in the table one have to be aware that the phases of the amplitudes $\beta$ include the kinematically dependent phases of the constant amplitudes $\alpha$ estimated at the lowest order in momentum.

\section{Comparison with weak resonance models}

The results from the ChPT fit allow to make some remarks of the validity of weak resonance models. In what follows we will consider weak deformation and factorization models [14]. Both models rely on the hypothesis that the strong chiral Lagrangian dominates the features of the $\Delta S=1$ effective lagrangian. Due to the saturation of the strong couplings of $O\left(p^{4}\right)$ by resonance exchange, the considered models can be also treated as models for the resonance contributions to the weak couplings. As it was shown in [14], the resonance model leads to a scale independent prediction $K_{2}-K_{3} \simeq 2.6 \times 10^{-9}$, which seems to be compatible with the values in Table 5 . A further assumption that only vector resonance contribute implies $K_{1}=K_{2}=0$ and $K_{3} \neq 0$. Even taking into account the scale dependencet of $K_{2}$, the vector resonance model is far from describing the data. Now lets consider the prediction

\footnotetext{
${ }^{5}$ the fit has been repeated by excluding the requirement for the smallness of the relative isospin phases

${ }^{6}$ the scale dependencies of the weak coupling can be found in 14]
} 


$$
-K_{1} \simeq K_{2}=K_{3} \simeq k_{f}\left(1.7 \times 10^{-9}\right),
$$

which comes from the weak deformation and the factorization models. The scale factor $k_{f}$ in (15) is equal to $1 / 2$ for the weak deformation model and is around unity for the factorization model. Contrariwise, the obtained values for the couplings $K_{2}$ and $K_{3}$ require $k_{f} \approx 4$. The relatively large uncertainties of the weak coupling constants and their sizable scale dependence can reduce this estimation by a factor of 2 , which unfortunately remains sufficiently far from the preferred by the factorization model value of $\approx 1$.

\section{Conclusions}

The applied ChPT approach establish certain relations between $K \rightarrow 2 \pi$ amplitudes and the constant and the linear amplitudes in $K \rightarrow 3 \pi$ decays. Moreover, the quadratic $K \rightarrow 3 \pi$ amplitudes are strongly correlated to the precisely measured constant and linear ones and therefore are independent of the weak coupling constants. The fit to the experimentally measured isospin amplitudes have shown that the chiral constraints are well satisfied not only for the $\Delta I=1 / 2$ amplitudes, but also for the $\Delta I=3 / 2$ ones. This fact leads to the conclusion of the validity of the chiral scheme in the description of the nonleptonic kaon decays.

In addition to these achievements, the presented analysis has two main disadvantages. The first one is that we have omitted the radiative corrections to $K \rightarrow 3 \pi$ amplitudes. As it was shown in [9], these corrections may introduce significant effects especially in the case of $K^{+} \rightarrow \pi^{+} \pi^{+} \pi^{-}$decay. The second disadvantage is neglecting the strong rescattering phases in the estimation of $K \rightarrow 3 \pi$ amplitudes. The latter is due to the fact that the experimental information in $\mathrm{CP}$ conserving $K \rightarrow 3 \pi$ decays is insufficient to provide these phases. From the other side, the real counterterms have been fitted to the assumed real isospin amplitudes and as a consequence the obtained values of the strong rescattering phases are suppressed. Therefore, it would be more desirable to fix the phases, for example, in precise timeinterferometry experiments [10] and to use the measurements as an input to the fitting procedure.

I am grateful to P.Hristov and L.Litov for useful discussions and comments. 


\section{References}

[1] A.Pich, Rept. Prog. Phys. 58, 563 (1995).

[2] J.Gasser and H.Leutwyler, Nucl. Phys. B250, 465 (1985).

[3] J.Kambor et al., Phys. Lett. B261, 496 (1991).

[4] A.Angelopoulos et al., Eur. Phys. J. C5, 389 (1998).

[5] V.Batusov et al., Nucl. Phys. B516, 3 (1998).

[6] R.Wanke for the NA48 collaboration, talk on "Recontres de Physique de la Vallée d'Aoste", La Thuile, 2001.

[7] D.Groom et al., Review of Particle Physics, Eur. Phys. J. C15, 1-878 (2000).

[8] T.Devlin and J.Dickey, Rev. Mod. Phys. 51, 237 (1979).

[9] A.Belkov et al., Phys. Part. Nucl. 26, 239 (1995).

[10] G.D'Ambrosio et al., Phys. Rev. D50, 5767 (1994); erratum, ibid. D51, 3975 (1995).

[11] J.Kambor et al., Nucl. Phys. B346, 17 (1990).

[12] J.Kambor et al., Phys. Rev. Lett. 68, 1818 (1992).

[13] J.Bijnens, G.Ecker and J.Gasser, The Second $D A \Phi N E$ Physics Handbook (Frascati, 1995).

[14] G.Ecker et al., Nucl. Phys. B394, 101 (1993). 
Table 1: Experimental data for the widths and the Dalitz plot slopes in $\mathrm{CP}$ conserving $K \rightarrow 3 \pi$ decays.

\begin{tabular}{|l|c|c|c|c|}
\hline Decay & $\Gamma\left(s^{-1}\right) \times 10^{-6}$ & $g \times 10^{1}$ & $h \times 10^{2}$ & $k \times 10^{2}$ \\
\hline \hline$K_{L} \rightarrow \pi^{+} \pi^{-} \pi^{0}$ & $2.43 \pm 0.04$ & $6.78 \pm 0.08$ & $7.6 \pm 0.6$ & $0.99 \pm 0.15$ \\
\hline$K_{L} \rightarrow \pi^{0} \pi^{0} \pi^{0}$ & $4.08 \pm 0.06$ & & $-0.50 \pm 0.23$ & \\
\hline$K^{+} \rightarrow \pi^{+} \pi^{+} \pi^{-}$ & $4.52 \pm 0.04$ & $-2.154 \pm 0.035$ & $1.2 \pm 0.8$ & $-1.01 \pm 0.34$ \\
\hline$K^{+} \rightarrow \pi^{0} \pi^{0} \pi^{+}$ & $1.40 \pm 0.03$ & $6.52 \pm 0.31$ & $5.7 \pm 1.8$ & $1.97 \pm 0.54$ \\
\hline$K_{S} \rightarrow \pi^{+} \pi^{-} \pi^{0}$ & $0.0036 \pm 0.0013$ & & & \\
\hline
\end{tabular}

Table 2: Values of the isospin amplitudes in units of $10^{-8}$, obtained from the phenomenological fit to the experimental data (first column) and from the fit within $O\left(p^{4}\right) \mathrm{ChPT}$ (second column).

\begin{tabular}{|c|c|c|}
\hline & Experiment & $O\left(p^{4}\right)$ \\
\hline \hline$a_{1 / 2}, \mathrm{keV}$ & $0.4665 \pm 0.0010$ & 0.4665 \\
\hline$a_{3 / 2}, \mathrm{keV}$ & $0.02116 \pm 0.00007$ & 0.02115 \\
\hline$\alpha_{1}$ & $92.12 \pm 0.34$ & 92.11 \\
\hline$\alpha_{3}$ & $-6.41 \pm 0.44$ & -6.97 \\
\hline$\beta_{1}$ & $-26.73 \pm 0.39$ & -26.76 \\
\hline$\beta_{3}$ & $-2.26 \pm 0.44$ & -2.17 \\
\hline$\gamma_{3}$ & $2.89 \pm 0.28$ & 2.98 \\
\hline$\zeta_{1}$ & $-0.38 \pm 0.19$ & -0.51 \\
\hline$\zeta_{3}$ & $-0.09 \pm 0.10$ & -0.008 \\
\hline$\xi_{1}$ & $-1.80 \pm 0.29$ & -1.66 \\
\hline$\xi_{3}$ & $0.17 \pm 0.15$ & 0.07 \\
\hline$\xi_{3}^{\prime}$ & $-0.57 \pm 0.41$ & -0.15 \\
\hline$\chi^{2} /$ n.d.f & $14.2 / 5$ & $13.9 / 14$ \\
\hline
\end{tabular}


Table 3: Values of the reduced one-loop amplitudes $a_{i}^{(8)}$ and $a_{i}^{(27)}$ for the renormalization scale $\mu=M_{\eta}$.

\begin{tabular}{|c|c|c|c|c|}
\hline & $\Re e a_{i}^{(8)}$ & $\Im m a_{i}^{(8)}$ & $\Re e a_{i}^{(27)}$ & $\Im m a_{i}^{(27)}$ \\
\hline \hline$A_{0}$ & 1.79 & 2.22 & -1.26 & -1.48 \\
\hline$A_{2}$ & - & - & -1.94 & 4.63 \\
\hline$\alpha_{1}$ & 2.31 & 1.00 & -1.21 & -0.67 \\
\hline$\alpha_{3}$ & - & - & 22.5 & 6.70 \\
\hline$\beta_{1}$ & -1.07 & 0.50 & 0.70 & 0.33 \\
\hline$\beta_{3}$ & - & - & -0.63 & -2.19 \\
\hline$\gamma_{3}$ & - & - & -3.58 & 1.12 \\
\hline$\zeta_{1}$ & -0.027 & 0.019 & 0.013 & -0.012 \\
\hline$\zeta_{3}$ & - & - & 0.035 & 0.066 \\
\hline$\xi_{1}$ & -0.115 & 0 & 0.088 & 0 \\
\hline$\xi_{3}$ & - & - & -0.126 & 0 \\
\hline$\xi_{3}^{\prime}$ & - & - & 0.294 & 0.430 \\
\hline
\end{tabular}

Table 4: Values of the strong counterterm coupling constants $L_{i}$ for the normalization scale $\mu=M_{\eta}$ in units of $10^{-3}$.

\begin{tabular}{|c|c|c|c|c|}
\hline$L_{1}$ & $L_{2}$ & $L_{3}$ & $L_{4}$ & $L_{5}$ \\
\hline \hline $0.6 \pm 0.3$ & $1.75 \pm 0.3$ & $-3.5 \pm 1.1$ & $0.0 \pm 0.5$ & $2.2 \pm 0.5$ \\
\hline
\end{tabular}


Table 5: Values of the redefined coupling constants $c_{2}^{\prime}, c_{3}^{\prime}$ and the counterterms $K_{i}$ in units of $10^{-9}$ obtained from full fit to the experimentally measured isospin amplitudes. The errors are from the experimentally measured amplitudes (first column of Table 2) and from the uncertainties in the strong couplings $L_{i}$ (Table 4 ), respectively.

\begin{tabular}{|c|c|}
\hline$c_{2}^{\prime} / F_{\pi}^{2}$ & $65.47 \pm 0.14$ \\
\hline$c_{3}^{\prime} / F_{\pi}^{2}$ & $-0.828 \pm 0.003$ \\
\hline$K_{2}$ & $6.9 \pm 0.1 \pm 2.2$ \\
\hline$K_{3}$ & $7.0 \pm 0.7 \pm 2.0$ \\
\hline$K_{5}$ & $-0.016 \pm 0.003 \pm 0.010$ \\
\hline$K_{6}$ & $-0.12 \pm 0.08 \pm 0.02$ \\
\hline$K_{7}$ & $-0.17 \pm 0.06 \pm 0.001$ \\
\hline
\end{tabular}

Table 6: The phases of the constant and linear isospin amplitudes.

\begin{tabular}{|c|c|c|c|c|c|}
\hline & $\alpha_{1}$ & $\alpha_{3}$ & $\beta_{1}$ & $\beta_{3}$ & $\gamma_{3}$ \\
\hline \hline phase & 0.07 & 0.09 & -0.12 & -0.09 & -0.03 \\
\hline
\end{tabular}

